7th International Workshop on Astronomy and

Relativistic Astrophysics (IWARA 2016)

International Journal of Modern Physics: Conference Series

Vol. 45 (2017) 1760074 (4 pages)

(C) The Author(s)

DOI: $10.1142 / S 2010194517600746$

\title{
Heuristic Approach on Anomalous Apsidal Precession of Planets
}

\author{
Abraão J. S. Capistrano, Joice A. M. Penagos \\ Universidade Federal da Integração Latino-Americana (UNILA) \\ Foz do Iguaçu, Paraná, 85867-970, Brazil \\ abraao.capistrano@unila.edu.br,bioastro@gmail.com \\ Manuel S. Alárcon \\ Departamento de Física, Universidad de los Andes \\ Cra 1 N 18 A - 12, 111711 Bogotá, Colombia \\ mf.sanchez17@uniandes.edu.co
}

Published 15 August 2017

\begin{abstract}
In this paper we study the anomalous movement of planet precession as compared with different observational data from Ephemerides of the Planets and the Moon (EPM2008 and EPM2011) and the Planetary and Lunar Ephemeris (INPOP10a). Using a heuristic methodology we obtain a very close results to observations.
\end{abstract}

Keywords: Anomalous Precession, Nearly-Newtonian Approximation, Ephemerides.

PACS numbers: 96.15.De, 95.30.Sf

\section{The Weyl Disk Conformastatic Solution}

We focus the present work on the description of the anomalous apsidal precession of planets. The effect of this precession is very small of order of -6 milliarcsecond as identified by E.V. Pitjeva ${ }^{1,2}$ with EPM2008 ephemerides, which suggested a retrograde precession of Saturn with relevant nonzero statistical significance. Several proposal have been made from deviations of standard classical gravity setup ${ }^{3}$ up to modifications of gravity itself including (or not) dark matter ${ }^{2}$. In this work, using the main results of the Weyl conformastatic solution ${ }^{4}$ in addition with a prior heuristic approach. To this matter, we compare our results to different observations on Ephemerides of the Planets and the Moon (EPM2008) ${ }^{1}$, (EMP2011) ${ }^{5,6}$ and on Planetary and Lunar Ephemeris (INPOP10a) ${ }^{7}$. To this matter, use the Weyl's

This is an Open Access article published by World Scientific Publishing Company. It is distributed under the terms of the Creative Commons Attribution 4.0 (CC-BY) License. Further distribution of this work is permitted, provided the original work is properly cited. 
conformastatic solution from Weyl's cylindrical symmetric line element given by ${ }^{8}$

$$
d s^{2}=e^{2(\lambda-\sigma)} d r^{2}+r^{2} e^{-2 \sigma} d \theta^{2}+e^{2(\lambda-\sigma)} d z^{2}-e^{2 \sigma} d t^{2},
$$

where $\lambda=\lambda(r, z)$ and $\sigma=\sigma(r, z)$. Since we are studying a movement of a test particle around the sun, we can consider the exterior gravitational field calculated in the circular border of thin cylindrical geometry, which is given by Einstein's vacuum equations

$$
\begin{aligned}
& -\lambda_{, r}+r \sigma_{, r}^{2}-r \sigma_{, z}^{2}=0, \\
& \sigma_{, r}+r \sigma_{, r r}+r \sigma_{, z z}=0 \\
& 2 r \sigma_{, r} \sigma_{, z}=\lambda_{, z},
\end{aligned}
$$

where the terms $(, r),(, z)$ and $(, r),(,, z)$ denote, respectively, the first and the second derivatives with respect to the variables $r$ and $z$. As shown in Weyl's original paper, the cylinder solution is diffeomorphic to a Schwarzschild's solution, hence the metric remains asymptotically flat ${ }^{8-12}$, which can be useful to astrophysics purposes. To this matter, we assume that the cylinder thickness $h_{0}$ is smaller than its radius $R_{0}$, i.e., $h_{0}<<R_{0}$. As a consequence, one can expand the coefficients $\lambda(r, z)$ and $\sigma(r, z)$ into a MacLaurin's series such that

$$
\begin{aligned}
& \sigma(r, z) \approx \sigma(r, 0)+\left.z \frac{\partial \sigma(r, z)}{\partial z}\right|_{z=0}+\left.z^{2} \frac{\partial^{2} \sigma(r, z)}{\partial z^{2}}\right|_{z=0}+\cdots, \\
& \lambda(r, z) \approx \lambda(r, 0)+\left.z \frac{\partial \lambda(r, z)}{\partial z}\right|_{z=0}+\left.z^{2} \frac{\partial^{2} \lambda(r, z)}{\partial z^{2}}\right|_{z=0}+\cdots
\end{aligned}
$$

and can be truncated in second order ${ }^{4}$. The conformastatic solution is such that $\lambda(r, z)=0$, and one can obtain the relevant coefficient $\sigma(r, z)$ as

$$
\sigma(r, z)=\frac{k_{0}}{2} \ln (r)-\frac{c_{0} r^{2}}{2}+a_{0} z+c_{0} z^{2} .
$$

As a result, one can find the expression ${ }^{4}$

$$
\delta \phi=\delta \phi_{s c h} \pm \beta_{0} \nu \eta^{*}
$$

where we denote $\delta \phi_{s c h}=\frac{6 \pi G M}{c^{2} \gamma\left(1-\epsilon^{2}\right)}$ that stands for standard Einstein's result for Schwarzschild solution, $\gamma$ denotes the semi-major axis and $\epsilon$ denotes the eccentricity of the orbits. The term $\nu$ is the keplerian mean motion given by $\sqrt{\frac{G M}{\gamma^{3}}}$. The sign \pm refers to the particle deviation to the plane of the orbits and the scale factor $\eta^{*}$ is defined by $\eta^{*}=(180 / \pi)(3600) T$, and $T$ is the number of revolution per century. For the perihelion advance, the $\beta_{0}$ parameter was found to be

$$
\beta_{0}=\epsilon^{4} \sqrt{1-\epsilon^{2}},
$$

restricted to the interval $[0,1]$ valid for all planets. It is important to note that eq.(8) can provide both advance and retrograde apsidal precession. 


\section{The Heuristic Approach}

Starting from considering the anomalous precession just a fraction of the apsidal perihelion precession advance, from eq.(8) we can write the following expression

$$
\delta \phi_{\text {anom }}= \pm \frac{\Delta(\delta \phi)}{\eta^{*} \nu}
$$

where $\delta \phi_{\text {anom }}$ is the anomalous precession and $\Delta(\delta \phi)$ denotes the percentage difference given by $\Delta(\delta \phi)=n\left(\delta \phi_{o b s}-\delta \phi_{s c h}\right)$. The term $\delta \phi_{o b s}$ refers to the perihelion value from observations and $n$ is the percentage number. The results are shown in table (01). In order to avoid error propagation, we explore the possibility to have a range to vary a percentage fraction, once we have a considerable difference between the studied observational data (e.g, in EPM2013 ${ }^{10}$ we do not have extraprecession). Our error margins were very small $<10^{-8}$ and they were omitted here. As it can be observed in table (03), the results are very close to observations as compared one-by-one. As a first prior, from Mercury to Saturn, to model the EPM2008 ephemerides we have the percentage range varying as $0.55<n(\%)<0.009$. Moreover, to EPM2011 ephemerides and INPOP10a, we have the percentage ranges varying as $50<n(\%)<0.01$ and $6.67<n(\%)<0.01$, respectively. This percentage enlargement is not surprising at all since we have larger margin errors in Jupiter and Saturn observations. The drawback of this approach is that it cannot show what is the tendency of the parameter $n$ regarding these three datasets. In table (01), it is shown a comparison between the values for anomalous apsidal precession $\delta \phi_{\text {anom }}^{a, b, c}$ of the planets in units of miliarcsec/century to the EPM2088 and EPM2011 ${ }^{5,6}$ and the INPOP10a planetary ephemerides ${ }^{7}$, respectively.

Table 1. Comparison between the values for anomalous apsidal precession.

\begin{tabular}{lccccrr}
\hline Object & $\delta \phi_{\text {anom }}^{a}$ & EPM2008 & $\delta \phi_{\text {anom }}^{b}$ & EPM2011 & $\delta \phi_{\text {anom }}^{c}$ & INPOP10a \\
\hline Mercury & -3.7945 & $-3.6 \pm 5.0$ & -2.0237 & $-2.0 \pm 3.0$ & 0.4047 & $0.4 \pm 0.6$ \\
Venus & -0.3878 & $-0.4 \pm 0.5$ & 2.6585 & $2.6 \pm 1.6$ & 2.6493 & $0.2 \pm 1.5$ \\
Earth & -0.1869 & $-0.2 \pm 0.4$ & 1.8694 & $0.19 \pm 0.19$ & -0.1669 & $-0.2 \pm 0.9$ \\
Mars & -0.1057 & $-0.10 \pm 0.5$ & -0.1939 & $-0.02 \pm 0.037$ & 0.3877 & $-0.04 \pm 0.15$ \\
Jupiter & n.a & n.a & 60.28 & $58.7 \pm 28.3$ & -48.0000 & $-41 \pm 42$ \\
Saturn & -5.7876 & $-6.0 \pm 2.0$ & -0.2829 & $-0.32 \pm 0.47$ & 0.1910 & $0.15 \pm 0.68$ \\
\hline
\end{tabular}

\section{Future Prospects}

Due to the high error bars of the data, it is necessary to understand the tendency of the fit parameter would be driven. As future prospects, we will use the LevenbergMarquardt algorithm to study the systematics error propagation. Work on this topic is currently in progress.

\section{Acknowledgements}

Joice A. M. Penagos thanks UNILA for the grant IC-Unila/EditalPRPPG2015-2016. 


\section{References}

1. E. V. Pitjeva, Ephemerides EPM2008: The Updated Models, Constants, Data, Paper Presented at Journées "Systémes de Référence Spatio-Temporels" and X LohrmannKolloquium, Dresden, Germany (2010). http://syrte.obspm.fr/jsr/journees2008/pdf/ (accessed on 20 May 2016).

2. L. Iorio, A.J. 137, 3615 (2009).

3. L. Acedo Galaxies 2, 466 (2014).

4. A. J. S. Capistrano, W. L. Roque, and R. S. Valada, Month. Not. Roy. Astron. Soc., 444, 1639 (2014).

5. E. V. Pitjeva and N. P. Pitjev, Mon. Not. R. Astron. Soc. 432, 3431 (2013).

6. N. P. Pitjev and E. V. Pitjeva, Astron. Lett. 39, 141 (2013).

7. A. Fienga et al., Celest. Mech. Dyn. Astron. 111, 363 (2011).

8. H. Weyl, Ann. Phys. (Berlin) 359, 117 (1917).

9. R. Gautreau, R. B. Hoffman, and A. Armenti, Il Nuovo Cimento B 7(1), 71 (1972).

10. E. V. Pitjeva, Solar Syst. Res. 47, 386 (2013).

11. N. Rosen, Rev. Mod. Phys. 21, 503 (1949).

12. H. Stephani et al., Exact Solutions of Einstein's Field Equations. (Cambridge: Cambridge University Press, 2003).

13. C. Misner et al., Gravitation (W. H. Freeman and Company, San Francisco, 1973). 